\title{
When is Pharmacogenetic Testing for Antidepressant Response Ready for the Clinic? A Cost-effectiveness Analysis Based on Data from the STAR*D Study
}

\author{
Roy H Perlis*, I,2, Amanda Patrick ${ }^{3}$, Jordan W Smoller' and Philip S Wang ${ }^{4}$ \\ 'Psychiatric Genetics Program, Center for Human Genetics Research and Department of Psychiatry, Massachusetts General Hospital and \\ Harvard Medical School, Boston, MA, USA; ${ }^{2}$ Bipolar Clinical and Research Program, Massachusetts General Hospital, Boston, MA, USA; ${ }^{3}$ Division \\ of Pharmacoepidemiology and Pharmacoeconomics, Department of Medicine, Brigham and Women's Hospital, Harvard Medical School, Boston, \\ MA, USA; ${ }^{4}$ National Institute of Mental Health, Bethesda, MD, USA
}

\begin{abstract}
The potential of personalized medicine to transform the treatment of mood disorders has been widely touted in psychiatry, but has not been quantified. We estimated the costs and benefits of a putative pharmacogenetic test for antidepressant response in the treatment of major depressive disorder (MDD) from the societal perspective. Specifically, we performed cost-effectiveness analyses using statetransition probability models incorporating probabilities from the multicenter STAR*D effectiveness study of MDD. Costs and qualityadjusted life years (QALYs) were compared for sequential antidepressant trials, with or without guidance from a pharmacogenetic test for differential response to selective serotonin reuptake inhibitors (SSRIs). Likely SSRI responders received an SSRI, whereas likely nonresponders received the norepinephrine/dopamine reuptake inhibitor bupropion. For a 40-year old with MDD, applying the pharmacogenetic test and using the non-SSRI bupropion for those at higher risk for nonresponse cost \$93520 per additional QALY compared with treating all patients with an SSRI first and switching sequentially in the case of nonremission. Cost per QALY dropped below $\$ 50000$ for tests with remission rate ratios as low as 1.5, corresponding to odds ratios $\sim$ I.8-2.0. Tests for differential antidepressant response could thus become cost effective under certain circumstances. These circumstances, particularly availability of alternative treatment strategies and test effect sizes, can be estimated and should be considered before these tests are broadly applied in clinical settings.

Neuropsychopharmacology (2009) 34, 2227-2236; doi:I 0. I038/npp.2009.50; published online 3 June 2009
\end{abstract}

Keywords: pharmacogenetics; major depressive disorder; cost effectiveness; serotonin

\section{INTRODUCTION}

Multiple antidepressant medications have demonstrated efficacy for the treatment of major depressive disorder (MDD). However, prospective comparisons and metaanalyses suggest little difference in efficacy between them. At the same time, many patients do not reach remission with initial antidepressant treatment, with consequences including greater functional impairment, greater likelihood of discontinuing treatment prematurely, and substantially increased medical costs associated with more chronic illness. It has been suggested that, by allowing patients to be matched to the treatment likely to be most effective for

*Correspondence: Dr RH Perlis, Department of Psychiatry, Harvard University, Massachusetts General Hospital, 15 Parkman St, WACC 812, Boston, MA 02114, USA, Tel: + 16177267426 , Fax: + 1617 724 3028, E-mail: rperlis@partners.org

Received 29 July 2008; revised 19 March 2009; accepted 27 March 2009 them, pharmacogenetic testing will provide an opportunity to improve depression treatment outcomes.

Recent studies have suggested that common genetic variations are associated with antidepressant response (Kim et al, 2006; McMahon et al, 2006; Perlis et al, 2008). Many of these results do not consistently replicate, do not address specificity of effect, or do not allow the estimation of the tests' performance in a general clinical population. Still, with larger clinical cohorts, these limitations are being overcome and the development of pharmacogenetic predictors of treatment response has become an active area of investigation.

Surprisingly, the question of when such testing will be suitable for clinical application, commonly raised by clinicians, has received minimal attention in psychiatry (Perlis et al, 2005). Nonetheless, at least two psychiatric pharmacogenetic tests are now commercially available, with others in development.

The Sequenced Treatment Alternatives to Relieve Depression $\left(\mathrm{STAR}^{\star} \mathrm{D}\right)$ study, which examined prospective 
outcomes in a very large cohort of patients receiving sequential antidepressant trials in a 'real-world' design, offers a unique opportunity to directly address the question of cost effectiveness (Rush et al, 2004). We utilized clinical and genetic data from $\operatorname{STAR}^{\star} \mathrm{D}$ to estimate the cost effectiveness of a recently reported pharmacogenetic test for antidepressant response that was replicated using a split-sample design (McMahon et al, 2006). Beyond examining the potential utility of that test as a 'base case', we developed a general-purpose model that allows the utility of any similar test to be estimated.

\section{METHODS}

We modeled a population of individuals in a current episode of MDD, using a 3-year time horizon and societal perspective - ie, examining outcomes over 3 years from the perspective of the costs and health benefits to society. We incorporated data from the clinical literature in a model to estimate the outcome of alternative diagnostic and treatment strategies for a typical patient beginning outpatient treatment for MDD (Figure 1). The treatment algorithm was based on the 'switch' arms of the STAR ${ }^{\star} \mathrm{D}$ study, with each treatment period lasting up to 12 weeks. Individuals are treated first with the selective serotonin reuptake inhibitor (SSRI) citalopram. Those who fail to remit are then switched to bupropion, followed by nortriptyline, and then by the combination of venlafaxine and mirtazapine.

We modeled the implementation of the test for SSRI responsiveness, either before any treatment (test-first) or after an initial treatment failure (test-second), compared to the 'no-test' condition (Figure 1). In the 'test-first' condition, those with a test result indicating greater likelihood of SSRI response are triaged to an SSRI, whereas those with a lesser likelihood are triaged to bupropion. In the 'testsecond' condition, those who fail an initial SSRI receive either a second SSRI or bupropion, based on test results. As outcomes with the atypical antidepressant bupropion or the SSRI sertraline as next-step treatments were similar in $\mathrm{STAR}^{\star} \mathrm{D}$ (Rush et al, 2006b), we also varied the treatment strategies, allowing individuals to receive SSRI or bupropion as first- or second-line treatment. For purposes of comparison, we also examined a no-test, bupropion-first/ citalopram-second strategy, to confirm that the test's potential benefit did not simply derive from shifting patients to a more effective and lower cost strategy.

We developed a state-transition model of transition probabilities in which patients could occupy distinct health states, including depressed (on or off treatment) and well (on or off treatment) (Simon et al, 2006). (For a review of the application of state-transition models in cost-effectiveness research in a related field, see Hsieh and Meng (2007)). We used a cohort simulation to track transitions between states representing the expected effects among patients of the alternate strategies (Supplementary Figure 1), with a cycle length of 3 months, corresponding to the duration of each treatment level in $\mathrm{STAR}^{\star} \mathrm{D}$. In other words, every 3 months, individuals can experience transition from one state to another. Models were constructed using the TreeAge Pro 2007 decision analysis program (TreeAge Software, Williamstown, MA).

\section{Transition Probabilities}

For each state in the state-transition model, there is an associated mortality rate describing probability of death in a given cycle. For depressed patients, this rate is calculated by adding the rate of suicide among depressed patients (O'Carroll et al, 1996) to age-adjusted all-cause mortality rates from US life tables (National Center for Health Statistics, 1998). For remitted patients, this is equal to the age-adjusted all-cause mortality rate. For sensitivity analyses, we used upper and lower bounds of the $95 \%$ confidence intervals around estimates of rates of death by suicide (Table 1).

Probabilities of remission, discontinuation, and recurrence were drawn directly from those reported in $S_{T A R}{ }^{*} \mathrm{D}$ for each treatment level (Rush et al, 2006a). Because STAR*D and prior meta-analysis (Salloum et al, 2005) did not identify significant differences in remission between SSRI and bupropion, values for bupropion and SSRI were set to be equal. Probabilities of recurrence following remission for treated and untreated

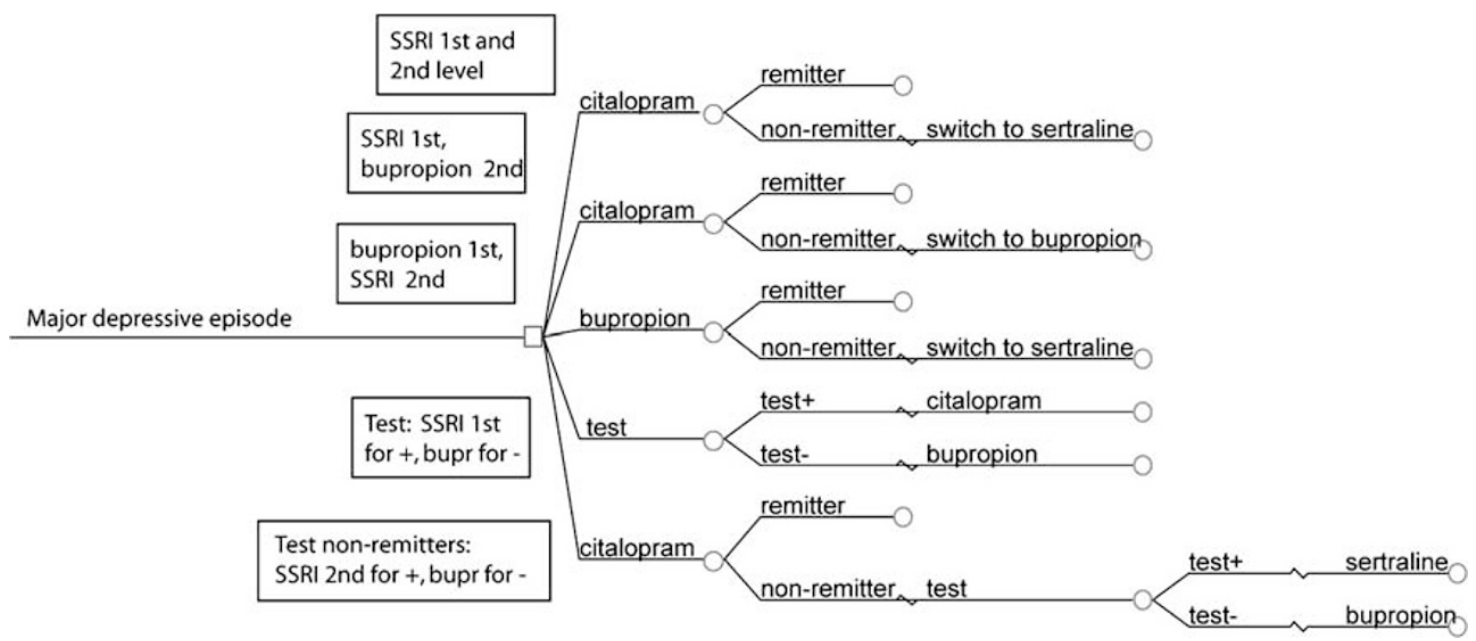

Figure I Decision analytic model for antidepressant treatment of major depressive disorder. It presents a schematic of the decision model used in this analysis. All patients begin in a major depressive episode. They may receive initial treatment with citalopram or bupropion, with or without treatment assignment based on the result of the genetic test. Individuals who fail to respond to initial treatment may receive sertraline or bupropion. 
Table I Parameters Used in the Base-Case and Sensitivity Analyses

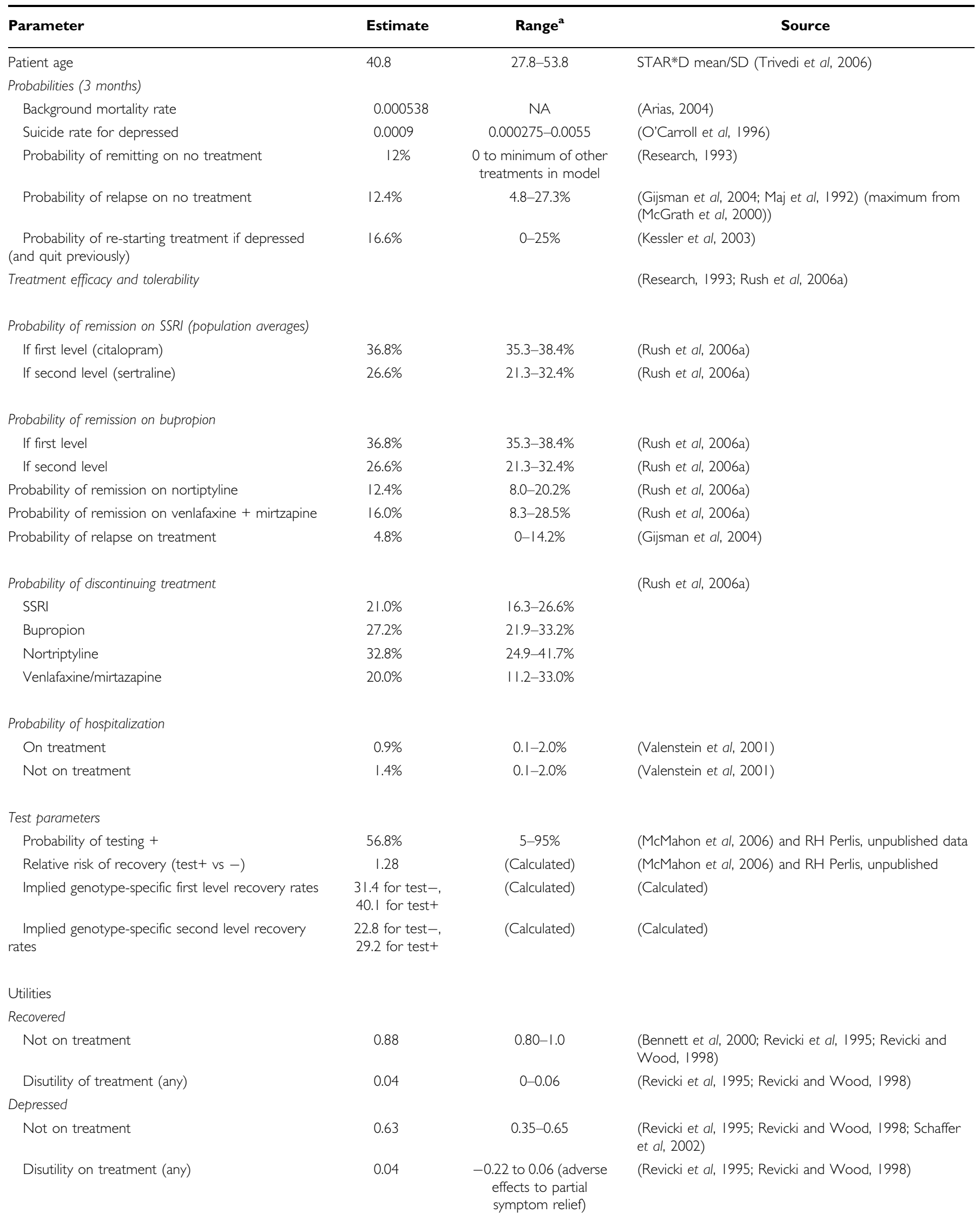


Table I Continued

\begin{tabular}{|c|c|c|c|}
\hline Parameter & Estimate & Range $^{\mathbf{a}}$ & Source \\
\hline \multicolumn{4}{|l|}{ Costs } \\
\hline \multicolumn{3}{|l|}{ Drug costs (3 month) } & \multirow{4}{*}{$\begin{array}{l}\text { (Anonymous, 2006) (Based on formulations closest in size } \\
\text { to median dose) }\end{array}$} \\
\hline SSRI (citalopram) & $\$ 251$ & $\$ 188-314$ & \\
\hline Bupropion & $\$ 442$ & $\$ 332-553$ & \\
\hline Nortiptyline & $\$ 248$ & $\$ 186-310$ & \\
\hline Genotyping test cost & $\$ 500$ & $\$ 0-1000$ & (Staples, 2007) \\
\hline Cost of hospitalizations due to depression & $\begin{array}{c}\$ 6889 \text { per } \\
\text { hospitalization }\end{array}$ & $\$ 5167-8622$ & (Agency for Healthcare Research and Quality, 2007) \\
\hline
\end{tabular}

${ }^{a}$ Except where specified, parameter ranges utilize $95 \%$ confidence intervals calculated from, or cited in, primary sources; for costs, parameter ranges utilize $\pm 25 \%$ of base-case value.

patients utilized data from a systematic review (Gijsman et al, 2004), with ranges incorporating additional long-term studies (Maj et al, 1992; McGrath et al, 2000).

\section{Utilities (Health Effects)}

Rather than simply considering whether subjects are alive or dead at each time point, the use of weights or utilities allows models to consider that different mood states may be associated with different quality of life (QOL). QOL weights for each mood and treatment state were drawn from the published literature and can range from 0 to 1 , with 1 representing 'perfect' QOL. Remitted depression was assigned a utility of 0.88 based on values reported in studies assessing this health state directly using standard gamble and time-trade-off techniques (Bennett et al, 2000; Revicki et al, 1995; Revicki and Wood, 1998). Remitted depression without prophylaxis received utilities of $0.86-$ 0.895 . These values are generally consistent with the utilities reported for 40-year olds in the Beaver Dam Health Outcomes Study (Fryback et al, 1993) and in more recent studies (Fryback et al, 2007; Sullivan et al, 2005). Untreated depression was assigned a utility of 0.63 , which was varied from 0.35 to 0.65 in sensitivity analyses (Revicki et al, 1995; Revicki and Wood, 1998). Utilities reported for depression vary widely from 0.09 for severe, untreated depression to 0.75 for mild depression (Schaffer et al, 2002); the base case was selected conservatively to reflect the prevalence of mild depression in outpatient populations, as well as so-called 'partial responders' who improve with treatment but do not remit. Treatment was assigned a disutility of 0.04 in our base case to reflect side effects associated with treatment, varied from a disutility of 0.06 (adverse effects - ie, worse QOL among individuals experiencing adverse effects and no symptomatic change) to a utility of 0.22 (partial symptom improvement without full remission) in sensitivity analyses.

\section{Costs}

The following direct medical costs were included in the basecase model: outpatient treatment (medication management) visits, hospitalization for severe depression, and antidepressant medications. Direct costs due to suicide were not included to avoid double counting, as hospitalization rates already include hospitalizations for suicide. All costs were inflated to 2006 US dollars using the medical care component of the consumer price index (CPI-M) (Gold et al, 1996). Drug costs were calculated from average wholesale generic price for the minimum number of pills necessary for median doses drawn from $\mathrm{STAR}^{*} \mathrm{D}$.

As recommended by the Panel on Cost-effectiveness in Health and Medicine (Weinstein et al, 1996), indirect costs such as those related to lost productivity and career opportunities were not included in the analysis. Such disease effects are likely to be captured in the utility weights assigned by patients to health states such as depression, and would therefore be double counted if included as costs as well.

\section{Pharmacogenetic Testing Parameters}

Although a number of pharmacogenetic tests are under active development, we focused on a single nucleotide polymorphism in the serotonin $2 \mathrm{~A}$ receptor (HTR2A) gene, which was associated with citalopram response in the largest antidepressant pharmacogenetic study to date and replicated in a split-sample design (McMahon et al, 2006). That publication did not report effect size for association with remission, and utilized a definition of remission in which individuals with intermediate response phenotypes (ie, significant improvement without remission) were omitted. We therefore obtained primary genotypic data from that study and calculated pertinent test parameters, including probability of a positive test in a mixed-ethnicity cohort $(56.8 \%)$ and remission rate ratio $(1.28$; $95 \%$ CI, $1.13-$ 1.42) for those with and without at least one copy of the 'risk' allele at rs7997012 in HTR2A. As the true mechanism of effect for this variation is not known, we assumed a dominant model of effect because it demonstrated stronger association than under a recessive model (RH Perlis, unpublished data). For one-way sensitivity analysis, we assumed a range of probabilities of a positive test between 0.05 and 0.95 . As the overall remission rate in the STAR ${ }^{\star} \mathrm{D}$ 
clinical cohort was somewhat less than that observed in the genetic cohort (McMahon et al, 2006), we used weighted averages to recalculate remission rates assuming overall remission rates $(36.8 \%$ for level 1 and $26.6 \%$ for level 2 ) equivalent to that in the clinical rather than genetic cohort. Of note, as the error rates with modern genotyping techniques are $\ll 1 \%$, and the reported test performance already accounts for such methodological errors, we did not further correct for this source of error. For the one-way sensitivity analyses, as a primary purpose of this model was to examine the function of test parameters in pharmacogenetic test performance, we examined the effect of remission rate ratios ranging from 1 (ie, no effect) to 2 (doubling of remission rate), corresponding to odds ratios for remission between 1 and 2.9 under our base assumptions for remission probability and allele frequencies. We considered effects in terms of remission rate ratios because this value is easily calculated from results in clinical trials and is not dependent on allele frequency (or probability of a positive test).

\section{Discounting}

We discounted all costs and health effects at an annual rate of $3 \%$ for the base case, with sensitivity analyses performed between 0 and $5 \%$. This standard practice in costeffectiveness analysis sets current costs as being worth more than those occurring in the future, reflecting the opportunity cost of spending money now (rather than, eg, investing it elsewhere) (Weinstein et al, 1996).

\section{RESULTS}

\section{Base-Case Analysis}

For a 40-year old with MDD, the SSRI as first- and secondline strategy was both cheaper and more effective than all other no-test conditions (Table 2). This finding was driven by the lower cost and lower treatment discontinuation rate associated with SSRI treatment compared to bupropion treatment. Compared to this strategy of treating all patients with an SSRI as first- and second-line therapy, the strategy of testing patients first and initiating those testing negative on bupropion cost an additional $\$ 505.50$ per patient but provided an additional 0.0054 quality-adjusted life years (QALYs), yielding an incremental cost-effectiveness ratio (ICER) of $\$ 93520$ (Table 2). ICER refers to the marginal (increase in) cost divided by the marginal (increase in) effectiveness, compared to the next most costly optionhow much additional cost would be required for each 1-year increase in QALYs. The strategy of testing following an initial treatment failure was eliminated by extended dominance: relative to the common alternative of no testing, the strategy of testing patients first had a lower ICER, providing better value per dollar spent. (This is an example of extended dominance because testing first is more costly than testing following a treatment failure. If it were less costly, testing following a treatment failure would be eliminated through simple dominance as a more costly and less effective strategy. For additional examples, see UDoVAHER Center.)

\section{Sensitivity Analyses}

In one-way sensitivity analyses, we examined the impact of varying individual model parameters bearing on costs, probabilities, and utility of mood and treatment states. In most one-way sensitivity analyses, the ICER for testing fell in the \$80000-100000 per QALY range. As expected, the cost of the test itself had a large effect on cost effectiveness; as test cost varied from $\$ 100$ to 1000 , ICER ranged from $\$ 19152$ to 186029 . When test cost was set to $\$ 0$, the least costly strategy remains two SSRI trials, without testing. The other nondominated strategy is the 'test-first' approach, which costs an additional $\$ 5.50$ and adds 0.0054 QALYs for an ICER of $\$ 1010$. Costs of medication management visits, hospitalizations, and pharmacotherapies in general did not meaningfully impact ICER. In the latter case, this lack of difference is primarily attributable to the availability of generic preparations for the primary treatment options. The comparative cost of bupropion $v s$ citalopram did impact which treatment-first option was favored, but with little meaningful effect on the ICER of testing.

We next examined the effects of varying test parameters or clinical cohorts. When we varied the response risk ratio over its 95\% confidence interval (1.13-1.42), the ICER for testing decreased from $\$ 218000$ to 59000 per QALY. We also considered scenarios where the genotype-specific remission rates are the same as in the base case, but the allele frequency is different. This circumstance might arise, eg, if a test identified in one ethnic group is applied in another ethnic group. In this case, the test's cost effectiveness is greatest as the probability of a positive test approaches $\sim 52 \%$, the point at which the effectiveness of citalopram-first and bupropion-first strategies are equivalent. The cost per QALY is less than $\$ 100000$ for probability of a positive test between 36 and 59\% (Figure 2). When the prevalence of a positive result is either very high or very low, the choice of initial treatment strategy is more clear-cut and testing provides relatively little improvement in overall remission rates. At prevalence of 5 or $95 \%$, the ICER of testing exceeds $\$ 750000$ per QALY.

We also explored the circumstances under which a different genetic test predicting SSRI response might be cost effective. To do this, we held the overall level 1 and level 2 SSRI and bupropion response rates constant at 36.8 and $26.6 \%$ but varied the strength of the genotype/SSRI response association and the prevalence of the different genotypes in a two-way analysis (ie, an analysis showing the effects of varying both parameters simultaneously) (Supplementary Figure 1). The benefit of genotyping is greatest when the prevalence of the two genotypes is approximately equal and when the absolute difference in response rates between the positive and negative test groups is the greatest. Under the base-case assumptions, at a 'willingness to pay' of $\$ 50000$ per QALY, the testing strategy can be cost effective for ratios of remission between positive and negative test subjects as low as 1.5 , provided the probability of a positive test is around $50 \%$; this corresponds to an odds ratio of $\sim 1.9$.

For the primary analyses, efficacy of bupropion and SSRI were constrained to be the same as initial treatment, consistent with meta-analysis that fail to find significant efficacy differences between these treatments as first-line antidepressant options (Salloum et al, 2005). In sensitivity 
Table 2 Results of Cost-Effectiveness Analysis

Base-case analysis

Incremental values

\begin{tabular}{|c|c|c|c|c|c|}
\hline Strategy & Cost $(\$)$ & Effectiveness & Cost $(\$)$ & Effectiveness & ICER \\
\hline No test-SSRI as level I and 2 & 3380 & 2.119 & & & \\
\hline No test-SSRI then bupropion & 3477 & 2.118 & & & Dominated $^{\mathrm{a}}$ \\
\hline No test-bupropion then SSRI & 3481 & 2.117 & & & Dominated \\
\hline Test if nonresponse & 3765 & 2.121 & & & Extended dominance $^{a}$ \\
\hline Test (+ gets SSRI then SSRI) & 3890 & 2.124 & 510.00 & 0.0054 & $\$ 93520$ \\
\hline
\end{tabular}

ICER, incremental cost-effectiveness ratio; SSRI, selective serotonin reuptake inhibitor; bupr, bupropion.

Incremental values here are presented for nondominated strategies only and are relative to the preceding nondominated strategy.

${ }^{a}$ Strategies are dominated if there is a competing strategy that is more effective and less costly. They are subject to extended dominance, sometimes referred to as weak dominance, if its incremental cost-effectiveness ratio is greater than, and its effectiveness less than, a competing strategy. For example, here 'test if nonresponse' is eliminated through extended dominance because its ICER is greater than the 'test, give test-positive subjects SSRI followed by another SSRI trial', and its effectiveness is less. (For further examples, see UDoVAHER Center).
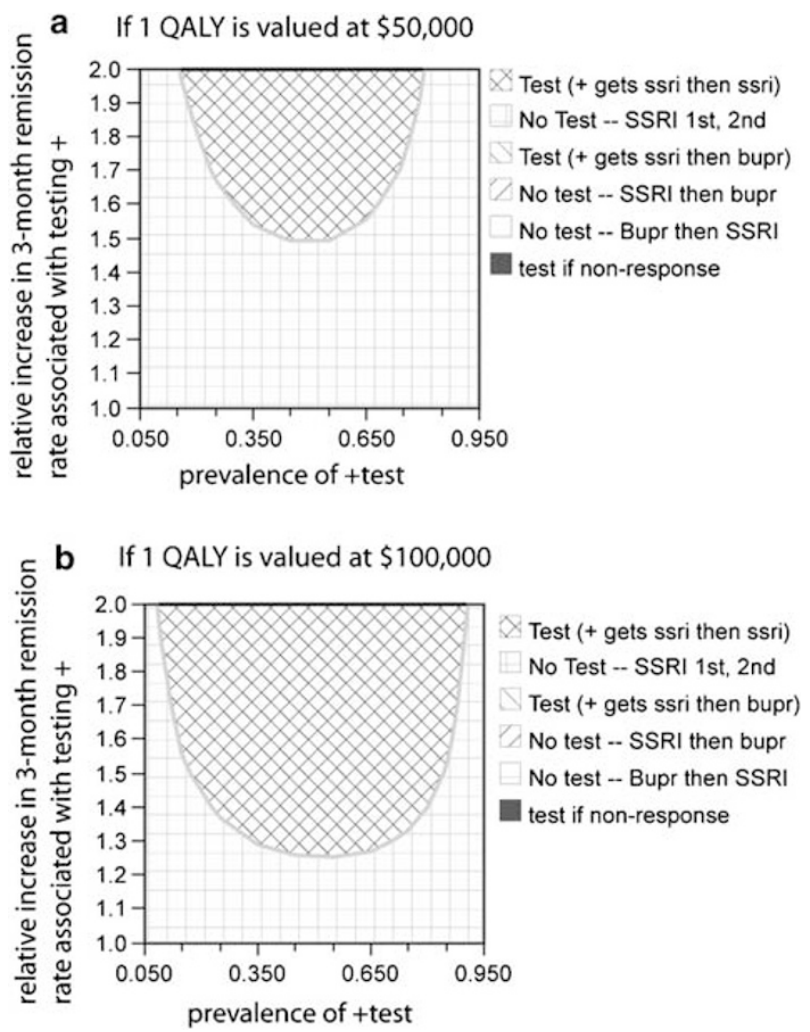

Figure 2 Two-way sensitivity analysis of the prevalence of a positive test result and the strength of association between test result and SSRI response. The top panel assumes a willingness to pay off $\$ 50000$ per quality-adjusted life year (QALY). The bottom panel increases this value to $\$ 100000$ per QALY. For each value of the prevalence of positive test and test effect size, an optimal strategy can be found by identifying the corresponding region in the graph and matching the color of that region to the color-coded key. SSRI, selective serotonin reuptake inhibitor; bupr, bupropion.

analysis, their efficacy was allowed to vary independently. As expected, this variation primarily impacts the relative cost effectiveness of a bupropion-first $v s$ SSRI-first strategy. However, in the extreme case where SSRI treatment is modeled as being substantially more efficacious, the benefit of bupropion treatment for negative test subjects is reduced and therefore the benefit of testing to identify these subjects is also reduced. When the level 1 bupropion remission rate was reduced to $35.3 \%$, testing (which triages negative test patients to bupropion) became less effective and the ICER for this strategy increased to $\$ 145000$ per QALY. Parameters of antidepressant treatment response otherwise had little impact on the model.

Finally, we varied the utility of mood states. As expected, when the disutility of depression increased (ie, the discomfort of remaining depressed is considered to be greater), the value of testing increased, and vice versa. Notably, reducing the utility of untreated depression to 0.35 reduced the ICER to $\$ 54128$ per QALY with base-case assumptions, suggesting that testing may be more cost effective in a more severely depressed population. In threeway sensitivity analysis, examining prevalence of a positive test, remission rate ratio for positive $v s$ negative test, and utility of the depressed state (Supplementary Figure 2), for a depression utility of 0.35 , testing ICER is below $\$ 50000$ if the likelihood of a positive test is $\sim 50 \%$ and remission rate ratio (for positive $v s$ negative test) is greater than 1.3.

\section{DISCUSSION}

Most genetic association studies conclude with a statement about how positive findings could be used to improve clinical outcomes, though cost effectiveness of genetic tests has received remarkably little attention in psychiatry (Perlis et al, 2005). With our base-case assumptions, utilizing a large-scale effectiveness study intended to mimic clinical practice, the incremental cost effectiveness of a putative pharmacogenetic test is $\$ 93520$ per QALY relative to the next best strategy of using an SSRI as first- and second-level treatment for all subjects. Although there is no accepted threshold below which interventions should be funded, one widely cited number, based on the cost effectiveness of dialysis in chronic renal failure patients covered by Medicare, is $\$ 50000$ per QALY (Winkelmayer et al, 
2002). It has been noted that few interventions with cost-effectiveness ratios exceeding $\$ 100000$ per QALY receive funding (Laupacis et al, 1992). Within psychiatry, a recent cost-effectiveness analysis suggested that a simple depression care program for employees led to an ICER of $\$ 20000$ per QALY (Simon et al, 2006), consistent with other primary care quality-improvement programs yielding ratios less than $\$ 50000$ (Simon et al, 2001). Relative to these numbers, the ICER for the genetic test, with our base-case assumptions, would not be considered cost effective. Of course, as genotyping rapidly becomes a commodity, the cost of testing would likely fall substantially. In the extreme, where testing is free, the cost per QALY is $\sim \$ 1000$, well within the range considered to be cost effective. Notably, the magnitude of difference between QALYs resulting from the strategies examined is modest, and below the threshold suggested by some authors to represent clinically meaningful differences (Kaplan et al, 1993). On the other hand, given the prevalence and costliness of MDD, even modest differences in outcomes bear consideration by policymakers. In the subset of patients whose treatment is changed by testing, the initial response rate is increased by $5 \%$. With a 0.25 QALY difference between a year of depression and a year of remission, this is arguably a clinically meaningful improvement.

With base-case assumptions, we found that a pharmacogenetic test for antidepressant response could only be considered cost effective for tests with odds ratios $\geqslant 1.5$. Multiple potential strategies could be applied by clinical researchers to identify such cost-effective tests. First, incorporating multiple informative loci will likely be necessary to achieve this threshold. Recent genome-wide association studies of antidepressant response indicate that individual loci are likely to exert only modest effects (Hamilton, 2007), so any pharmacogenetic test would likely need to incorporate multiple informative loci to achieve an adequate odds ratio. Second, more effective tests could incorporate other putative clinical predictors such as those identified in the $\operatorname{STAR}^{\star} \mathrm{D}$ study (Trivedi et al, 2006). Addition of clinical predictors would simply be reflected in better test performance (ie, greater effect sizes).

An alternate strategy would rely on tests informative about multiple treatment strategies: rather than focusing solely on SSRIs, a test that was also informative about common alternative strategies could be more cost effective. To date, few antidepressant pharmacogenetic studies include such non-SSRI comparators and describe specific predictors for the alternate strategy.

Similarly, the incorporation of predictors of adverse effects could offer another strategy for designing costeffective tests. Although modern antidepressants are quite safe and generally well tolerated, many patients do discontinue treatment prematurely. A number of recent reports suggest that it may be possible to predict specific adverse effects (Laje et al, 2007; Perlis et al, 2003, 2007).

Our results underscore the importance of understanding pharmacogenetic test performance in the population in which it is being applied. Although this is true in general for any test, it becomes particularly important given the known wide variation in allele frequencies between racial groups (The International HapMap Consortium, 2003). Apart from individual studies in Southeast Asian or Latino populations
(Kim et al, 2006; Wong et al, 2006), the vast majority of association studies for antidepressant responsiveness focus on Caucasians. Notably, the 'beneficial' allele frequency for the test considered here is less prevalent among African Americans (McMahon et al, 2006). Our results demonstrate that the cost effectiveness of such tests is critically dependent upon the effect size, and test probabilities, in the target population, suggesting that more representative cohorts will be required to determine the true utility of pharmacogenetic tests.

We note several caveats in interpreting our base-case results. Most importantly, our estimates rely on numerous assumptions about model parameters that are imprecise and likely to vary across clinical settings. However, a strength of this study is that it closely follows results of one of the largest antidepressant-effectiveness studies completed to date. Not only was that study designed to mirror clinical practice, but it took place in both primary care and specialty psychiatric clinics, suggesting our results can be informative about 'real-world' treatment of MDD (Rush et al, 2004). Many of the parameters not drawn from $\operatorname{STAR}^{\star} \mathrm{D}$ were previously utilized in a cost-effectiveness model of an employer-based depression intervention (Wang et al, 2006), which was later validated in a prospective study (Wang et al, 2007). This model can thus be understood in terms of 'how might $\mathrm{STAR}^{\star} \mathrm{D}$ outcomes have differed if initial treatment assignment was determined by a genetic test', assuming a standard set of next-step interventions. Of note, our results likely underestimate the 'true' cost effectiveness of the intervention because, as with most such analyses, we do not include the costs to caregivers or other family members (Weinstein et al, 1996).

We emphasize that, although we utilized an existing genetic finding as our base case, the general model can be applied to any pharmacogenetic test of antidepressant response. The code for this model is available at (http:// pngu.mgh.harvard.edu/perlis); simply substituting the appropriate test parameters allows cost effectiveness of that test to be estimated. The first marketed pharmacogenetic test to be advocated for antidepressant prescribing is actually one that examines cytochrome P450 variation (Somanath et al, 2002), though it has previously been suggested that such a test is likely to have little impact on general antidepressant prescribing (Perlis, 2007). Similarly, a serotonin transporter promoter insertion/deletion polymorphism is the genetic variation most often associated with antidepressant responsiveness, albeit in small cohorts (Serretti et al, 2006). However, the specificity of its effect is not well characterized, and the largest cohort to date did not detect an association with treatment response, though incorporating an additional polymorphism did identify some association with overall citalopram tolerability ( $\mathrm{Hu}$ et al, 2007). Therefore, we focused on the HTR2A variation because it was replicated in a split-sample design and exerts a well-defined impact on response. We note that, even if it represents a true association, its effect size is almost certainly less than that estimated here, based on the phenomenon of the 'winner's curse', or regression to the mean. Future pharmacogenetic tests will almost certainly incorporated multiple markers drawn from genome-wide association studies, but the basic principles of our model can be applied regardless of the type or scale of the genetic test. 
We also made the simplifying assumption that the HTR2A-based test is not informative about response to other (non-SSRI) treatments. Although this appears to be the case in STAR ${ }^{\star} \mathrm{D}$ and other cohorts (Perlos et al, 2009), these effects have not been fully characterized. In general, this assumption would yield an optimistic estimate in the base case, and underscores the need to understand not simply predictors of nonresponse to a given treatment, but the specificity of such predictors, before their clinical application. That is, it will be important to characterize not only predictors of differential response to a single treatment, but also the effects of such predictors on response to alternative treatment strategies.

Third, we included two primary types of states in our state-transition model, 'depressed' and 'well', either on- or off-antidepressant. The gradation of treatment responses in depression is well known - based on $\mathrm{STAR}^{\star} \mathrm{D}$, roughly onethird of patients improve with treatment but do not reach symptomatic remission (Trivedi et al, 2006). The effects of including individuals who improve but do not remit in treatment among the 'depressed' state would be to dilute or decrease the disutility of depression, rendering our model overly conservative. On the other hand, the significant impact of continued depressive symptoms even among those who improve with treatment is well documented (Wells et al, 2007).

A further simplification was the requirement that nonremitters have their treatment switched rather than augmented (Fava, 2001). In routine outpatient settings, augmentation is generally a much less common strategy, particularly in primary care settings. A survey of clinicians suggested that substantial variation exists in their preference for treatment sequence (Petersen et al, 2002), perhaps because before STAR ${ }^{\star} \mathrm{D}$ there was little controlled data bearing on the efficacy of augmentation in general (Fava, 2001).

Notwithstanding these caveats, our results suggest a means for evaluating future pharmacogenetic tests in psychiatry. To our knowledge, only one previous report addressed the value of such psychiatric tests in terms of cost effectiveness; in that study, we found that a test for clozapine responsiveness could be cost effective under certain conditions (Perlis et al, 2005). As with pharmacotherapy, determining the true cost effectiveness of a diagnostic intervention will require either large randomized prospective studies or retrospective assessment of large clinical population. With the substantial public interest in personalized medicine, pressure will be great to quickly translate tests to clinical practice. Our results suggest that the cost effectiveness of these tests can be modeled in a straightforward fashion, allowing necessary test parameters and integration with treatment algorithms to be carefully considered before implementation.

\section{ACKNOWLEDGEMENTS}

This work was supported by NIMH K23-MH67060, NARSAD and the Sydney R Baer, Jr Foundation, and the Bowman Family Foundation (Dr Perlis) and NIMH R01MH6194 (Dr Wang). No pharmaceutical company was involved in any way in initiating, conducting, or supporting this research.

\section{DISCLOSURE/CONFLICT OF INTEREST}

\section{Roy Perlis}

Research Support: Eli Lilly \& Company

Advisory/Consulting: AstraZeneca, Bristol-Myers Squibb, Eli Lilly \& Company, Pfizer Inc., Proteus

Speaking: AstraZeneca, Bristol-Myers Squibb, Eli Lilly \& Company, GlaxoSmithKline, Pfizer Inc.

Equity Holdings: Concordant Rater Systems, LLC

Royalty/patent, other income: Concordant Rater Systems, LLC

Jordan Smoller

Dr Smoller has received honoraria from Hoffman-La Roche, Inc., and has served on an advisory board for Roche Diagnostics Corporation. He has received research funding from the National Institute of Mental Health and the National Alliance for Research in Schizophrenia and Depression.

The other authors have no financial competing interests to disclose.

\section{REFERENCES}

Agency for Healthcare Research and Quality (2007). Testing for cytochrome p450 polymorphisms in adults with non-psychotic depression treated with selective serotonin reuptake inhibitors (SSRIs). Evidence report/technology assessment 146. Accessed at www.ahrq.gov/downloads/pub/evidence/pdf/cyp450/cyp450.pdf. [this is a white paper].

Anonymous (2006). Drug Topics Red Book, 108th edn. Medical Economics: Montvale, NJ.

Arias E (2004). United States Life Tables. National Center for Health Statistics: Hyattsville, MD.

Bennett KJ, Torrance GW, Boyle MH, Guscott R, Moran LA (2000). Development and testing of a utility measure for major, unipolar depression (McSad). Qual Life Res 9: 109-120.

Centers for Medicare and Medicaid Services (2004). Physician fee schedule payment amount file national/carrier. Available at http://www.cms.hhs.gov/PhysicianFeeSched/01_overview.asp. Accessed on 1 October 2007.

Fava M (2001). Augmentation and combination strategies in treatment-resistant depression. J Clin Psychiatry 62(Suppl 18): 4-11.

Fryback DG, Dasbach EJ, Klein R, Klein BE, Dorn N, Peterson K et al (1993). The Beaver Dam Health Outcomes Study: initial catalog of health-state quality factors. Med Decis Making 13: 89-102.

Fryback DG, Dunham NC, Palta M, Hanmer J, Buechner J, Cherepanov D et al (2007). US norms for six generic healthrelated quality-of-life indexes from the National Health Measurement study. Med Care 45: 1162-1170.

Gijsman HJ, Geddes JR, Rendell JM, Nolen WA, Goodwin GM (2004). Antidepressants for bipolar depression: a systematic review of randomized, controlled trials. Am J Psychiatry 161: 1537-1547.

Gold MR, Siegel JE, Russell LB, Weinstein MC (eds) (1996). CostEffectiveness in Health and Medicine. Oxford University Press: New York.

Grembowski DE, Martin D, Patrick DL, Diehr P, Katon W, Williams B et al (2002). Managed care, access to mental health specialists, and outcomes among primary care patients with depressive symptoms. J Gen Intern Med 17: 258-269.

Hamilton S (2007). Pharmacogenetics in Psychiatry meeting. New York, NY.

Hsieh MH, Meng MV (2007). Decision analysis and Markov modeling in urology. J Urol 178: 1867-1874. 
Hu XZ, Rush AJ, Charney D, Wilson AF, Sorant AJ, Papanicolaou GJ et al (2007). Association between a functional serotonin transporter promoter polymorphism and citalopram treatment in adult outpatients with major depression. Arch Gen Psychiatry 64: 783-792.

Kaplan RM, Feeny D, Revicki DA (1993). Methods for assessing relative importance in preference based outcome measures. Qual Life Res 2: 467-475.

Kessler RC, Berglund P, Demler O, Jin R, Koretz D, Merikangas KR et al (2003). The epidemiology of major depressive disorder: results from the National Comorbidity Survey Replication (NCS-R). JAMA 289: 3095-3105.

Kim H, Lim SW, Kim S, Kim JW, Chang YH, Carroll BJ et al (2006). Monoamine transporter gene polymorphisms and antidepressant response in Koreans with late-life depression. JAMA 296: 1609-1618.

Laje G, Paddock S, Manji H, Rush AJ, Wilson AF, Charney D et al (2007). Genetic markers of suicidal ideation emerging during citalopram treatment of major depression. Am J Psychiatry 164: 1530-1538.

Laupacis A, Feeny D, Detsky AS, Tugwell PX (1992). How attractive does a new technology have to be to warrant adoption and utilization? Tentative guidelines for using clinical and economic evaluations. CMAJ 146: 473-481.

Maj M, Veltro F, Pirozzi R, Lobrace S, Magliano L (1992). Pattern of recurrence of illness after recovery from an episode of major depression: a prospective study. Am J Psychiatry 149: 795-800.

McGrath PJ, Stewart JW, Petkova E, Quitkin FM, Amsterdam JD, Fawcett J et al (2000). Predictors of relapse during fluoxetine continuation or maintenance treatment of major depression. J Clin Psychiatry 61: 518-524.

McMahon FJ, Buervenich S, Charney D, Lipsky R, Rush AJ, Wilson $\mathrm{AF}$ et al (2006). Variation in the gene encoding the serotonin $2 \mathrm{~A}$ receptor is associated with outcome of antidepressant treatment. Am J Hum Genet 78: 804-814.

National Center for Health Statistics (1998). Vital statistics of the United States, 1995. Volume II, Mortality, Part A, Section 6: life tables. DHHS Publication No (PHS) 98-1147, Hyattsville, MD, US Dept HHS.

O'Carroll PW, Berman AL, Maris RW, Moscicki EK, Tanney BL, Silverman MM (1996). Beyond the Tower of Babel: a nomenclature for suicidology. Suicide Life Threat Behav 26: 237-252.

Perlis RH (2007). Cytochrome P450 genotyping and antidepressants. BMJ 334: 759.

Perlis RH, Fijal B, Adams DH, Sutton VK, Trivedi MH, Houston JP (2009). Variation in catechol-O-methyltransferase is associated with duloxetine response in a clinical trial for major depressive disorder. Biol Psychiatry 65: 785-791.

Perlis RH, Ganz DA, Avorn J, Schneeweiss S, Glynn RJ, Smoller JW et al (2005). Pharmacogenetic testing in the clinical management of schizophrenia: a decision-analytic model. J Clin Psychopharmacol 25: 427-434.

Perlis RH, Mischoulon D, Smoller JW, Wan YJ, Lamon-Fava S, Lin $\mathrm{KM}$ et al (2003). Serotonin transporter polymorphisms and adverse effects with fluoxetine treatment. Biol Psychiatry 54: 879-883.

Perlis RH, Moorjani P, Fagerness J, Purcell S, Trivedi MH, Fava M et al (2008). Pharmacogenetic analysis of genes implicated in rodent models of antidepressant response: association of TREK1 and treatment resistance in the STAR $\left.{ }^{*}\right)$ D study. Neuropsychopharmacology 33: 2810-2819.

Perlis RH, Purcell S, Fava M, Fagerness J, Rush AJ, Trivedi MH et al (2007). Association between treatment-emergent suicidal ideation with citalopram and polymorphisms near cyclic adenosine monophosphate response element binding protein in the STAR ${ }^{\star} \mathrm{D}$ study. Arch Gen Psychiatry 64: 689-697.

Petersen T, Dording C, Neault NB, Kornbluh R, Alpert JE, Nierenberg AA et al (2002). A survey of prescribing practices in the treatment of depression. Prog Neuropsychopharmacol Biol Psychiatry 26: 177-187.

Research AfHCPa (1993). Depression in Primary Care. Vol 2. Treatment of Major Depression. Clinical Practice Guideline, No. 5. (AHCPR publication no. 93-0550.). U.S. Dept. of Health and Human Services: Rockville, MD.

Revicki DA, Brown RE, Palmer W, Bakish D, Rosser WW, Anton SF et al (1995). Modelling the cost effectiveness of antidepressant treatment in primary care. Pharmacoeconomics 8: 524-540.

Revicki DA, Wood M (1998). Patient-assigned health state utilities for depression-related outcomes: differences by depression severity and antidepressant medications. J Affect Disord 48: 25-36.

Rush AJ, Fava M, Wisniewski SR, Lavori PW, Trivedi MH, Sackeim HA et al (2004). Sequenced treatment alternatives to relieve depression $\left(\mathrm{STAR}^{\star} \mathrm{D}\right)$ : rationale and design. Control Clin Trials 25: $119-142$.

Rush AJ, Trivedi MH, Wisniewski SR, Nierenberg AA, Stewart JW, Warden D et al (2006a). Acute and longer-term outcomes in depressed outpatients requiring one or several treatment steps: a $\mathrm{STAR}^{\star}$ D report. Am J Psychiatry 163: 1905-1917.

Rush AJ, Trivedi MH, Wisniewski SR, Stewart JW, Nierenberg AA, Thase ME et al (2006b). Bupropion-SR, sertraline, or venlafaxine-XR after failure of SSRIs for depression. N Engl J Med 354: $1231-1242$.

Salloum IM, Cornelius JR, Daley DC, Kirisci L, Himmelhoch JM, Thase ME (2005). Efficacy of valproate maintenance in patients with bipolar disorder and alcoholism: a double-blind placebocontrolled study. Arch Gen Psychiatry 62: 37-45.

Schaffer A, Levitt AJ, Hershkop SK, Oh P, MacDonald C, Lanctot K (2002). Utility scores of symptom profiles in major depression. Psychiatry Res 110: 189-197.

Serretti A, Cusin C, Rausch JL, Bondy B, Smeraldi E (2006). Pooling pharmacogenetic studies on the serotonin transporter: a mega-analysis. Psychiatry Res 145: 61-65.

Simon GE, Savarino J, Operskalski B, Wang PS (2006). Suicide risk during antidepressant treatment. Am J Psychiatry 163: 41-47.

Simon GE, Von Korff M, Rutter CM, Peterson DA (2001). Treatment process and outcomes for managed care patients receiving new antidepressant prescriptions from psychiatrists and primary care physicians. Arch Gen Psychiatry 58: 395-401.

Somanath CP, Jain S, Reddy YC (2002). A family study of earlyonset bipolar I disorder. J Affect Disord 70: 91-94.

Staples J (2007). Considerations in the commercialization of genomic inventions. Available at http://people.genome.duke. edu/ merri034/CVGenomics/Slides/Staples.pdf. Accessed on 1 October 2007.

Sullivan PW, Lawrence WF, Ghushchyan V (2005). A national catalog of preference-based scores for chronic conditions in the United States. Med Care 43: 736-749.

The International HapMap Consortium (2003). The International HapMap Project. Nature 426: 789-796.

Trivedi MH, Rush AJ, Wisniewski SR, Nierenberg AA, Warden D, Ritz L et al (2006). Evaluation of outcomes with citalopram for depression using measurement-based care in $S T A R^{\star} D$ : implications for clinical practice. Am J Psychiatry 163: 28-40.

US Department of Veterans Affairs Health Economic Resource Center: http://www.herc.research.va.gov/resources/faq_a01.asp.

Valenstein M, Vijan S, Zeber JE, Boehm K, Buttar A (2001). The cost-utility of screening for depression in primary care. Ann Intern Med 134: 345-360.

Wang PS, Patrick A, Avorn J, Azocar F, Ludman E, McCulloch J et al (2006). The costs and benefits of enhanced depression care to employers. Arch Gen Psychiatry 63: 1345-1353.

Wang PS, Simon GE, Avorn J, Azocar F, Ludman EJ, McCulloch J et al (2007). Telephone screening, outreach, and care management for depressed workers and impact on clinical and work productivity outcomes: a randomized controlled trial. JAMA 298: 1401-1411. 
Weinstein MC, Siegel JE, Gold MR, Kamlet MS, Russell LB (1996). Recommendations of the panel on cost-effectiveness in health and medicine. JAMA 276: 1253-1258.

Wells KB, Schoenbaum M, Duan N, Miranda J, Tang L, Sherbourne C (2007). Cost-effectiveness of quality improvement programs for patients with subthreshold depression or depressive disorder. Psychiatr Serv 58: 1269-1278.
Winkelmayer WC, Weinstein MC, Mittleman MA, Glynn RJ, Pliskin JS (2002). Health economic evaluations: the special case of endstage renal disease treatment. Med Decis Making 22: 417-430.

Wong ML, Whelan F, Deloukas P, Whittaker P, Delgado M, Cantor RM et al (2006). Phosphodiesterase genes are associated with susceptibility to major depression and antidepressant treatment response. Proc Natl Acad Sci USA 103: 15124-15129.

Supplementary Information accompanies the paper on the Neuropsychopharmacology website (http://www.nature.com/npp) 